\title{
Automated Four-Point Probe Measurement of Nanowires Inside a Scanning Electron Microscope
}

\author{
Changhai Ru*, Member, IEEE, Yong Zhang*, Student Member, IEEE, Yu Sun, Senior Member, IEEE, Yu Zhong, \\ Xueliang Sun, David Hoyle, and Ian Cotton
}

\begin{abstract}
Nanomanipulation inside a scanning electron microscope (SEM) has been employed to maneuver and characterize nanomaterials. Despite recent efforts toward automated nanomanipulation, it is still largely conducted manually. In this paper, we demonstrate automated nanomanipulation inside an SEM for a well-structured nanomanipulation task via visual servo control and a vision-based contact-detection method using SEM as a vision sensor. Four-point probe measurement of individual nanowires is achieved automatically by controlling four nanomanipulators with SEM visual feedback. A feedforward controller is incorporated into the control system to improve response time. This technique represents an advance in nanomanipulation inside SEM and can be extended to other nanomanipulation tasks.
\end{abstract}

Index Terms-Automated nanomanipulation, contact detection, four-point probe, visual servo control.

\section{INTRODUCTION}

$\mathbf{U}$ SING electron microscopy as an imaging platform, nanomanipulation inside a scanning electron microscope (SEM) has been employed to maneuver and characterize the properties of nanomaterials. For instance, carbon nanotubes, In$\mathrm{GaAs} / \mathrm{GaAs}$ nanosprings, and silicon nanowires were deformed via a nanomanipulator inside an SEM to characterize their mechanical and/or electrical properties [1]-[6]. Through nanomanipulation, nanomaterials were also placed on microelectromechanical systems (MEMS) devices for tensile testing [7]-[11].

To date, SEM nanomanipulation has largely been performed manually by an operator using a joystick and/or a keypad while

Manuscript received May 9, 2010; accepted July 14, 2010. Date of publication August 12, 2010; date of current version July 8, 2011. This work was supported by the Natural Sciences and Engineering Research Council of Canada under a Discovery Grant and a Collaborative Research and Development Grant, by the Ontario Centers of Excellence under a Collaborative Research Grant, and by the Canada Research Chairs Program. The review of this paper was arranged by Associate Editor L. Dong.

* Shared first co-authorship. Contributed equally.

C. Ru is with the Robotics and Microsystems Center, Soochow University, Suzhou 215021, China. He was with the Advanced Micro and Nanosystems Laboratory, University of Toronto, Toronto, ON M5S 3G8, Canada.

Y. Zhang and Y. Sun are with Advanced Micro and Nanosystems Laboratory, University of Toronto, Toronto, ON M5S 3G8, Canada (e-mail: sun@mie.utoronto.ca).

Y. Zhong and X. Sun are with the Department of Mechanical and Materials Engineering, University of Western Ontario, ON N6A 5B9, Canada.

D. Hoyle and I. Cotton are with Hitachi High-Technologies Canada Inc., Toronto, ON M9W 6A4, Canada.

Color versions of one or more of the figures in this paper are available online at http://ieeexplore.ieee.org.

Digital Object Identifier 10.1109/TNANO.2010.2065236 constantly monitoring SEM images, which is time consuming, skill dependent, and often breaks end-effectors. As this technology becomes increasingly relevant for device prototyping [3], [12]-[16] as well as the for aforementioned material testing at the nanometer scale, progress is being made toward automated nanomanipulation in order to achieve high reliability, efficiency, and repeatability.

Existing nanomanipulators are driven by piezoelectric actuators and usually have only three translational degrees of freedom (DOF). Because of creep, drift, and hysteresis of piezoelectric actuators, open-loop control cannot suffice in precision for automatic nanomanipulation tasks, necessitating feedback control. Integrating position sensors (e.g., optical encoders or capacitive sensors) appears to be a straightforward solution and has been achieved for a few commercial nanomanipulators manufactured by, for example, SmarAct GmbH and Attocube Systems AG. However, the integration of high-resolution encoders increases the cost significantly; furthermore, sensor drift can be significant at the nanometer scale. Thus, visual feedback from SEM becomes essential for closed-loop control of nanomanipulators with/without encoders.

To visually obtain the $X Y$ position of an end-effector, visual tracking of the end-effector in a sequence of SEM images has been implemented using feature-based methods [17], [18] or correlation-based methods [17], [19]. For example, a rigidmodel-based visual tracking method was reported, which applies domain-specific constraints and was evaluated by tracking a microgripper inside an SEM [19]. Based on SEM visual tracking, visual servo control can be realized to control the in-plane position of the nanomanipulator, which, however, has not been reported in the literature.

Besides position control along $X Y$, precise positioning along the $z$-axis is also essential but more challenging since it is difficult to extract depth information from SEM visual feedback. To address this issue, a few techniques were proposed. The depth-from-focus method commonly used under an optical microscope was extended to SEM for a coarse estimate of the $Z$-position of an end-effector [20]. A touch sensor based on piezoelectric ceramics [20] and a shadow-based depth detection method [21] were used for a fine estimate of the $Z$-position. MEMS capacitive sensors for contact detection under optical microscopes [22] can also be used inside an SEM. However, the precisions of these methods have not been quantified.

In addition, stereoscopic SEM images can be generated by tilting the electron beam [23], which requires the installation of specialized hardware and needs more thorough studies in order to be used for automated nanomanipulation. Tilting the 


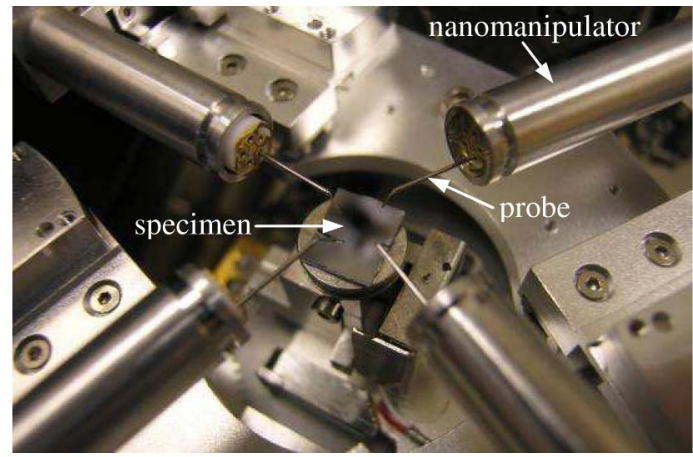

Fig. 1. Four nanomanipulators with probes installed for manipulation inside an SEM.

specimen can also be used for SEM stereoscopy [24], but has not been evaluated for nanomanipulation purposes. In summary, a convenient technique is needed which is capable of precisely estimating $Z$ positions or detecting contact between an endeffector and a substrate.

In this paper, we demonstrate fully automated nanomanipulation inside an SEM by performing a well-structured nanomanipulation task for probing the electrical properties of individual nanowires. Four-point probe measurement of nanomaterials has been reported using scanning tunneling microscopy inside an SEM [25]-[27], using four-probe devices [28]-[31], and using focused ion beam deposition or electron beam lithography to pattern electrodes [32], [33]. Nonetheless, existing techniques require expensive equipment and lack flexibility; moreover, they all entail tedious trial-and-error manual operation.

Our system, in this paper, controls four nanomanipulators inside SEM to realize an automated four-point probe measurement on individual nanowires lying on a substrate. Nanowires as well as nanoprobes installed on the nanomanipulators are visually recognized. The $X Y$ positions of the nanoprobes are controlled via image-based visual servo control with a feedforward controller. Their contact with the substrate is also detected via visual feedback. Current-voltage $(I-V)$ data of tin oxide $\left(\mathrm{SnO}_{2}\right)$ nanowires are obtained after the nanoprobes positioned on the nanowires.

\section{AUTOMATED NANOMANIPULATION INSIDE AN SEM}

\section{A. Nanomanipulation System}

A nanomanipulation system (Zyvex S100) is integrated into an SEM (Hitachi S-4000) by mounting its head assembly onto the specimen stage of the SEM. The head assembly, as shown in Fig. 1, is composed of four quadrants of three-DOF nanomanipulators, each of which is composed of a coarse-positioning stage and a fine-positioning unit. The coarse-positioning stage contains three identical piezoelectric slip-stick motors, each of which has a travel of $12 \mathrm{~mm}$ with $100-\mathrm{nm}$ resolution. The fine-positioning unit contains a piezoelectric tube having travel ranges of $10 \mu \mathrm{m}$ along the axis of the tube and $100 \mu \mathrm{m}$ along each of the two transverse directions with 5-nm nominal resolution.
The nanomanipulators do not have integrated position sensors for either coarse or fine positioning, as in most commercial and academic nanomanipulation systems. In Fig. 1, each nanomanipulator has a tungsten probe installed as an end-effector for interacting with the specimen placed at the center of the specimen stage. The probes have direct electrical connections to an interface located outside the SEM for applying or measuring electrical signals. Before loading the probes into the nanomanipulators, the probes are chemically cleaned to remove the native tungsten oxide using $\mathrm{KOH}$ solutions and $\mathrm{HF}$. After the cleaning procedure, the probe tips are 150-200 $\mathrm{nm}$ in diameter.

$\mathrm{SnO}_{2}$ nanowires being probed in this paper are synthesized for use as anode materials in Li-ion batteries. The nanowires are prepared by the chemical vapor deposition (CVD) method in a horizontal quartz tube. Sn powder is chosen as the starting material and is loaded in a ceramic boat. The ceramic boat is placed in the center of a quartz tube mounted in an electric furnace. A silicon wafer with a Au film of 5-nm thickness is placed close to the starting powder. An Ar flow is introduced into the furnace as the gas carrier. The furnace is heated up to $700{ }^{\circ} \mathrm{C}$, which is maintained for $2 \mathrm{~h}$. Finally, the furnace is cooled down to the room temperature under the Ar atmosphere. The nanowires are scratch removed and placed on $\mathrm{SiO}_{2}$-covered silicon substrate. Consequently, the nanowires lie directly on the substrate surface, which constitutes a well-structured manipulation condition and makes automated probing possible.

\section{B. Recognition of Probes and Nanowires}

When the four probes and one or multiple nanowires are in the field of view [see Fig. 2(a)], they are visually recognized and identified [see Fig. 2(b)]. The contours of the objects in the image are recognized through a sequence of low-pass Gaussian filtering, adaptive thresholding, and morphological operations. The probes and nanowires are distinguished by comparing areas surrounded by the contours, since nanowires have smaller areas. The four probes are distinguished from each other by comparing the positions of the centroids of their contours, and the positions of their tips are determined as either the highest or lowest point of a probe's contour. The target positions on the nanowire for probing are determined relative to the positions of the rightmost and leftmost points of the nanowire's contour, according to desired separations between the probe tips during measurements.

\section{Feature Tracking}

Visual tracking provides motion/position information of the probes in the image frame as position feedback for vision-based contact detection and visual servo control. The sum-of-squareddifferences (SSDs) algorithm is employed to track the tip of a probe. The system first conducts visual recognition in a frame of image $I_{1}$ to obtain the coordinate $\left(x_{1}, y_{1}\right)$ of the probe tip. A rectangular patch of the image containing the probe tip is recorded as a template. In subsequent frames, the SSD measure is calculated for each possible displacement $(\Delta x, \Delta y)$ within a 


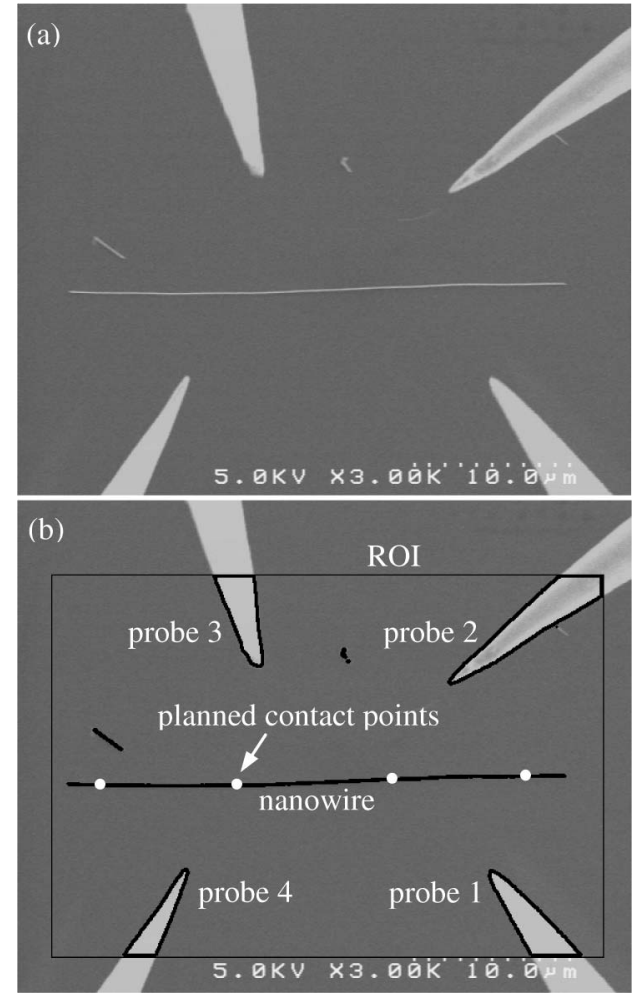

Fig. 2. Visual recognition of probes and nanowires from SEM visual feedback. (a) Four probes and nanowires. (b) Probes and nanowires are recognized from image processing.

search window. Specifically, in the $k$ th frame

$$
\begin{array}{r}
\operatorname{SSD}(\Delta x, \Delta y)=\sum_{i, j \in N}\left[I_{k}\left(x_{k-1}+\Delta x+i, y_{k-1}+\Delta y+j\right)\right. \\
\left.-I_{k-1}\left(x_{k-1}+i, y_{k-1}+j\right)\right]^{2}
\end{array}
$$

where $\left(x_{k-1}, y_{k-1}\right)$ is the coordinate of the probe tip in the $(k-1)$ th frame of image $I_{k-1}$. The displacement $(\Delta x, \Delta y)$ producing the minimum SSD value is considered to be the displacement of the probe. The system simultaneously tracks all the four probes using the SSD algorithm.

To achieve a subpixel-tracking resolution, eight neighboring pixels as well as the selected pixel are used to fit a curved surface in terms of their SSD values. The pixel coordinate corresponding to the valley of the curved surface is used to determine the displacement of the feature [34].

\section{Vision-Based Contact Detection}

Manipulation of a nanoobject lying on a substrate requires knowledge on relative vertical positions between the endeffector and the object/substrate. When a probe is moved downward, the system detects the contact between the probe tip and the substrate via a vision-based contact detection method, which was extended from a previous method we developed for optical microscopy [35]. This method makes use of the phenomenon that a downward-moving probe slides on the surface of the substrate after contact is established, as illustrated in Fig. 3.

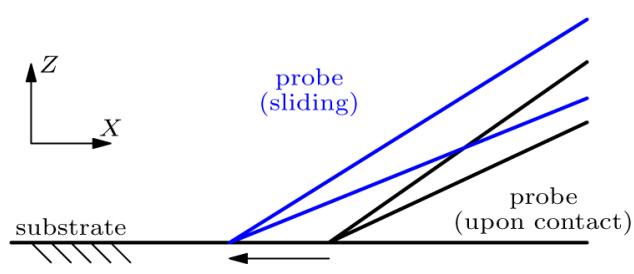

Fig. 3. Principle of vision-based contact detection: probe slides on the substrate after contact establishment.

Inside an SEM, when a nanomanipulator moves, a probe along its $z$-axis to approach a substrate, the position of the probe in the image frame also moves along a certain direction, due to the perspective projection model of the SEM [19]. When the probe contacts the substrate and begins to slide, an abrupt shift in the moving direction of the probe in the image frame is recognized as the contact point. Therefore, by monitoring the occurrence of this phenomenon, visually tracking a downwardmoving probe is capable of detecting the contact between the probe and the substrate.

\section{E. Visual Servo Control With a Feedforward Controller}

To achieve closed-loop positioning of a nanomanipulator in the $X Y$ plane, visual tracking of the end-effector is used to provide position feedback for a visual servo controller. Visual feedback from SEM has a low sampling frequency [typically $<15$ frames per second (fps)]. This issue was previously addressed by selectively scanning a smaller region of interest [18], [19]. However, the method requires the installation of new hardware for accessing the scan controller of the SEM.

Without altering a standard SEM configuration, we add a feedforward controller in the visual control system, as shown in Fig. 4. The feedforward controller contains a mathematical model [36], [37] accounting for the hysteresis of the piezoelectric nanomanipulator, such that the probe can be quickly moved to the vicinity of a target position without depending on visual feedback. The PID controller in the image-based visual servo control system then brings the probe tip precisely to the target position.

In order to obtain the parameters in the mathematical model, and hence, the feedforward controller, voltages are incrementally applied to each axis of the actuator and then incrementally released with the corresponding displacements in the image frame recorded by the visual-tracking algorithm. As an example, the characterization result of the $x$-axis of the piezoelectric tube of one of our four nanomanipulators at the magnification of 3,000× is shown in Fig. 5. The applied voltage is denoted by $u$, with its lowest and highest values denoted by $U_{0}$ and $U_{n}$. The ascending and descending curves are denoted by functions $P_{n}(u)$ and $D_{n}(u)$, and are respectively fitted with a fourthorder polynomial. If the piezoelectric actuator is at any point on the ascending (descending) curve, and the applied voltage is monotonically increasing (decreasing), the output displacement is $P_{n}(u)\left(D_{n}(u)\right)$.

However, if the applied voltage starts to decrease (increase) when the actuator is on the ascending (descending) curve, the actuator will stray away from that curve due to hysteresis. In 


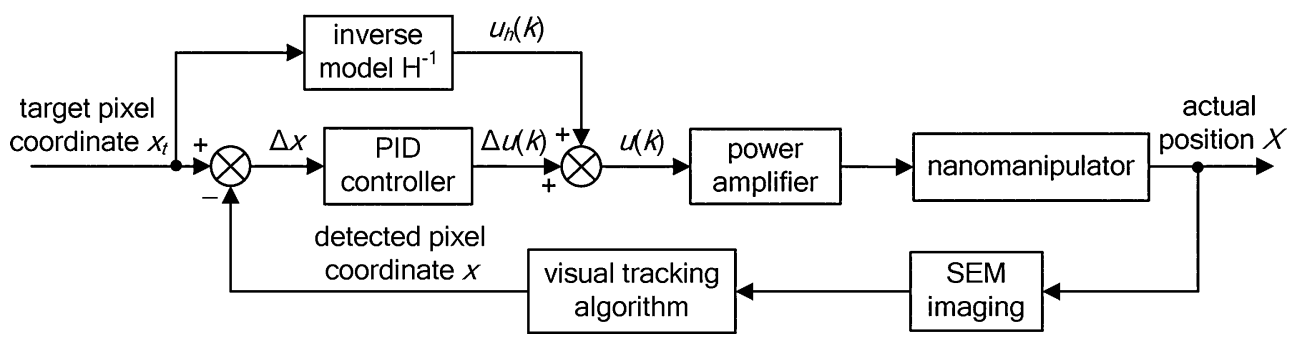

Fig. 4. SEM-image-based visual servo control system incorporating a feedforward controller. One axis is shown as an example.

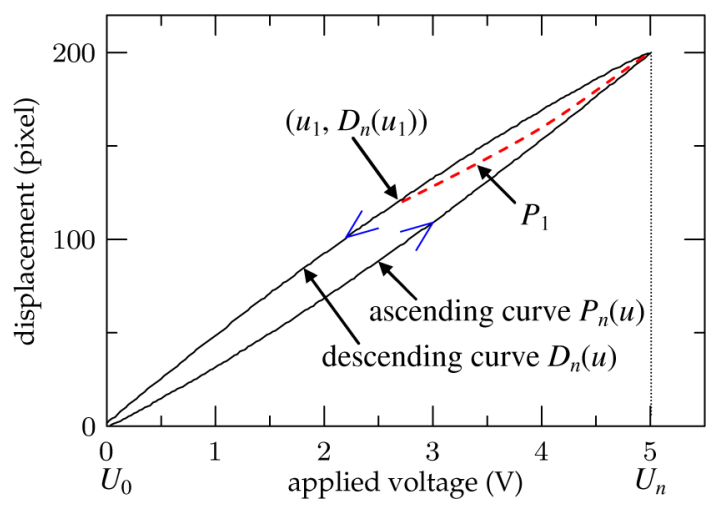

Fig. 5. Characterization of a piezoelectric actuator for feedforward control within the visual servo control system.

that case, a mathematical model is used to calculate the output displacement. When the applied voltage increases from point $\left(u_{1}, D_{n}\left(u_{1}\right)\right)$ on the descending curve [see Fig. 5], the output displacement (dashed curve in Fig. 5) is [36]

$P_{1}(u)=k \cdot P_{n}\left(m \cdot\left(u-U_{n}\right)+U_{n}\right)+D_{n}\left(U_{1}\right)-k \cdot P_{n}\left(U_{0}\right)$

where $k=\left(D_{n}\left(U_{n}\right)-D_{n}\left(u_{1}\right)\right) /\left(D_{n}\left(U_{n}\right)-D_{n}\left(U_{0}\right)\right) \quad$ and $m=\left(U_{n}-u_{1}\right) /\left(U_{n}-U_{0}\right)$.

When the actuator is on the descending curve and a certain displacement is desired, the corresponding applied voltage is calculated via the Newton-Raphson method to invert $D_{n}(u)$ or the mathematical model (2), as the output of the feedforward controller. A similar mathematical model is used for the scenario, where the applied voltage starts to decrease while the actuator is on the ascending curve to calculate the output of the feedforward controller.

\section{F. Overall Process of Automated Nanomanipulation}

The four probes are first brought into the field of view under a proper magnification using the coarse-positioning stages, after which only the fine-positioning units are controlled to move the probes. The probes and nanowires are visually recognized. The longest nanowire is selected for testing, and four target positions on the nanowire are determined. The system moves the probes downward to establish their contact with the substrate through vision-based contact detection. After contact detection, the probes are positioned at a certain height (e.g., $200 \mathrm{~nm}$ ) above the substrate, ready for subsequent in-plane movement.

The four piezoelectric tubes are actuated in their respective $x$ and $y$ axes to obtain parameters for their mathematical models

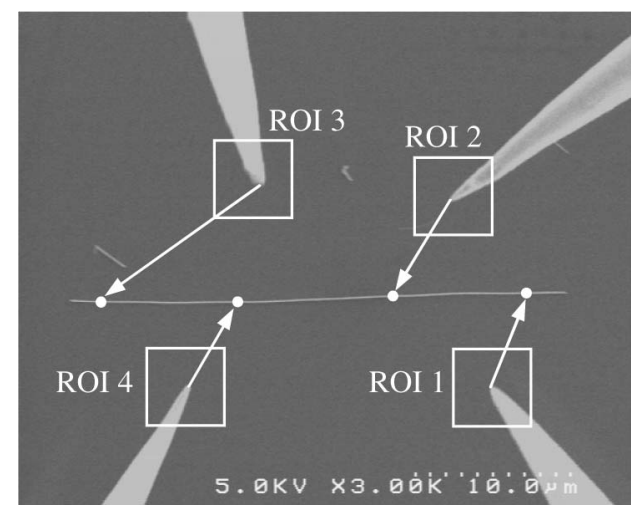

Fig. 6. Four probes simultaneously move to their target positions on a nanowire by feedforward visual servo control.

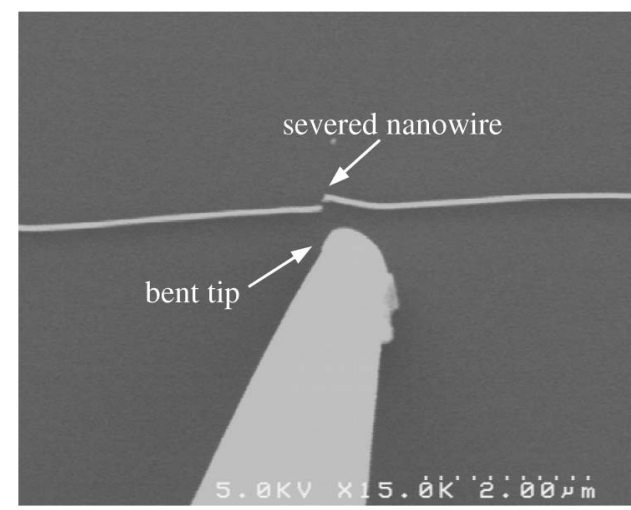

Fig. 7. Manual operation often causes probe tip damage and sometimes inadvertently severs a nanowire.

to be used in the feedforward controllers. Through visual servo control with a feedforward controller, the probes are simultaneously moved toward their target positions on the nanowire [see Fig. 6]. Then the probes move downward to land on the nanowire for measurements. The separation distance between the inner pair of probes can be automatically adjusted by repositioning the probes for measuring the electrical properties of different lengths of the nanowire.

\section{EXPERIMENTAL RESULTS AND DISCUSSIONS}

\section{A. Manual Operation}

For comparisons between automated nanomanipulation and manual operation, a nanomanipulator was manually controlled to perform the task. An experienced operator used a joystick and/or a program via mouse clicking to control actuation 
TABLE I

QUANTIFICATION OF SEM-IMAGE DRIFT AND NOISE

\begin{tabular}{c|c|c|c|c|c|c|c}
\hline \multirow{2}{*}{$\begin{array}{c}\text { magnification } \\
\text { (pixel size) }\end{array}$} & $\begin{array}{c}30,000 \times \\
(6.6 \mathrm{~nm} / \mathrm{pixel})\end{array}$ & $\begin{array}{c}10,000 \times \\
(20 \mathrm{~nm} / \mathrm{pixel})\end{array}$ & $\begin{array}{c}5,000 \times \\
(40 \mathrm{~nm} / \mathrm{pixel})\end{array}$ & $\begin{array}{c}3,000 \times \\
(66 \mathrm{~nm} / \mathrm{pixel})\end{array}$ & $\begin{array}{c}1,500 \times \\
(133 \mathrm{~nm} / \mathrm{pixel})\end{array}$ & $(330 \mathrm{~nm} / \mathrm{pixel})$ \\
\hline \multirow{2}{*}{$\begin{array}{c}\text { drift and noise } \\
\text { (pixel) }\end{array}$} & $x$ & 4.46 & 2.97 & 2.20 & 1.23 & 0.77 & 0.65 \\
\cline { 2 - 9 } & $y$ & 4.42 & 1.56 & 1.38 & 1.14 & 0.68 & 0.69 \\
\hline \multirow{2}{*}{$\begin{array}{c}\text { noise } \\
\text { (pixel) }\end{array}$} & $x$ & 1.27 & 0.97 & 0.72 & 0.68 & 0.77 & 0.60 \\
\cline { 2 - 9 }
\end{tabular}

voltages for a piezoelectric tube while monitoring the imaging screen. When approaching and contacting the substrate, the probe tip is often bent [see Fig. 7] before the operator realizes that the contact has been established, since it is difficult for a human operator to promptly perceive the initial contact between the probe tip and the substrate even at high magnifications due to the drift and noise in real-time SEM imaging.

Aside from probe damage, manually controlling a probe to probe a nanowire is also time consuming and can be destructive to the nanowire. Bringing the probe tip to a desired location takes much time since the $x$ and $y$ motions must be carefully coordinated. When the probe contacts and then is lifted off the nanowire, the probe sometimes inadvertently severs the nanowire, as shown in Fig. 7, since the contact between the probe tip and the nanowire is difficult to accurately determine by naked eyes observing noisy SEM imaging.

\section{B. Evaluation of Drift and Noise in SEM Imaging}

Drift and noise are inherently present in SEM imaging in the fast-scanning mode, both of which affect the performance of visual tracking. SEM image noise refers to random variations of pixel values and arises from a few sources. Image drift refers to the movement of the entire image, such as from electron beam drift, charge drift on a specimen, and/or electromagnetic interferences from the environment.

In order to quantify the magnitudes of image drift and noise, we used the subpixel visual-tracking method to track stationary features on a substrate. Table I summarizes standard deviations for different magnifications at the highest scanning rate of $13 \mathrm{fps}$. Besides showing the combinatorial effect of image drift and noise, Table I also shows the effect of image noise alone, which was obtained by simultaneously tracking two stationary features in the image to remove the effect of image drift. It can be seen from Table I that at higher magnifications, image drift and noise are more significant and are generally smaller along the $y$-direction than along the $x$-direction.

Since the effect of image drift can be eliminated, image noise is the primary source of errors in visual tracking and automated nanomanipulation, and therefore, of primary concern. In Table I, the image noise at the magnification of $3000 \times$ is approximately 0.68 pixel along the $x$-axis, which corresponds to $45 \mathrm{~nm}$ considering the pixel size of $66 \mathrm{~nm} /$ pixel. It can also be observed from

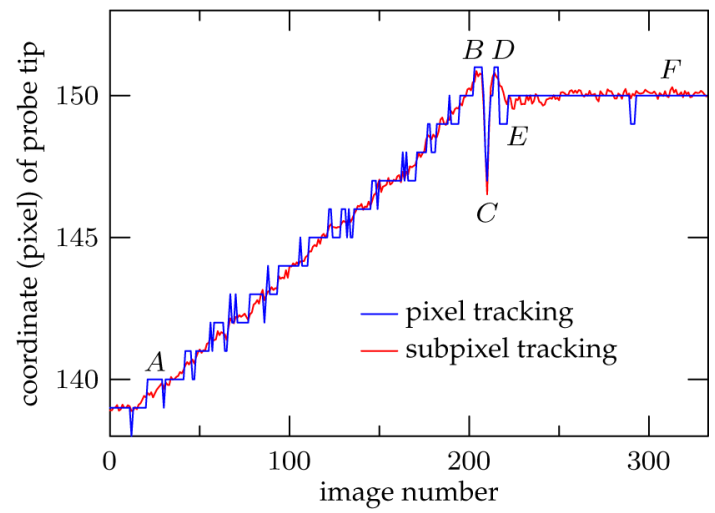

Fig. 8. Automated contact detection and subsequent $Z$-positioning of a probe via pixel- and subpixel-visual tracking.

Table I that although a higher magnification generally gives rise to higher image noise in pixels, the noise level in terms of nanometers is actually reduced at increasing magnifications. Thus, for manipulating nanoobjects smaller than $45 \mathrm{~nm}$, a higher magnification than $3,000 \times$ is necessary. Since the nanowires in this study have diameters ranging from $70 \mathrm{~nm}$ to $100 \mathrm{~nm}$, the $3,000 \times$ magnification was sufficient in terms of tracking resolution, and hence, automated probing requirements.

While image noise can be reduced by choosing slow-scanning modes of the SEM, lower frame rates are inadequate for automated nanomanipulation relying on the SEM visual feedback for closed-loop control.

\section{Contact-Detection Results}

Contact detection was performed in an area of the substrate where there were no nanowires or contaminants below the probe to ensure contact-detection accuracy. The system moved a probe downward at a constant speed. Meanwhile, the position of the probe tip in the image frame along its moving direction was monitored using visual tracking. As an example, Fig. 8 shows the results of automated contact detection and subsequent $Z$ positioning for one of the piezoelectric tubes via both pixel- and subpixel-tracking methods.

Each of the two data curves in Fig. 8 is comprised of five characteristic segments, representing five different stages of the process. First, in segment AB, the pixel coordinate of the probe 


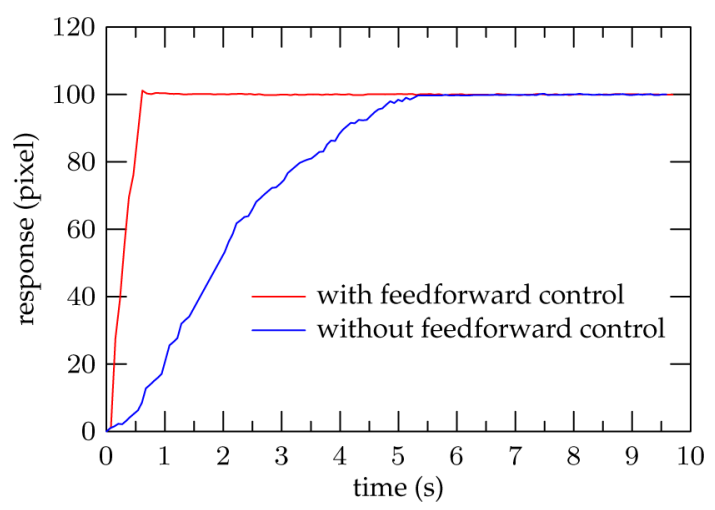

Fig. 9. Step responses of the visual servo control system for one axis of a piezoelectric tube with and without feedforward control.

tip in the image frame increases, corresponding to the stage that the probe tip approaches the substrate prior to contact. Second, in segment $\mathrm{BC}$, the pixel coordinate quickly decreases after point $\mathrm{B}$, which represents that the probe is sliding forward on the substrate surface after contact. At point $\mathrm{C}$, contact is determined by the system to have occurred since the pixel coordinate has decreased by five pixels, which is the preset threshold in consideration of the image noise level. Thereby at point $\mathrm{C}$ the probe is stopped from descending, and the initial contact point is determined to be point $\mathrm{B}$.

Third, in segment CD, the pixel coordinate increases again, which corresponds to the stage that the probe tip is sliding back as the probe is being raised. Fourth, in segment DE, the pixel coordinate decreases, which represents that the probe tip is ascending after having been lifted off the substrate. Fifth, in segment EF, the pixel coordinate remains unchanged within a margin of 0.6 pixel, which represents that the probe is positioned at a height above the substrate. By comparing the two curves in Fig. 8, it can be seen that the subpixel-tracking method demonstrates a superior resolution for processing noisy SEM images.

\section{Performance of Visual Servo Control}

A step signal (the desired displacement in pixels) was input to the visual servo control system. The responses of the system to a 100-pixel-step input with and without using the feedforward controller are shown in Fig. 9. It can be seen that the use of the feedforward controller shortens the rise time from 3.51 to 0.44 $\mathrm{s}$, and the settling time from 5.23 to $0.61 \mathrm{~s}$. The $X Y$ positioning accuracy was determined to be $42 \mathrm{~nm}$ at $3000 \times$ magnification. The much faster response is because the feedforward controller incorporates the mathematical model of the piezoelectric actuator and does not rely on any feedback. Low-frame-rate SEM visual feedback is only used after the probe tip is within the vicinity of the target position, for fine positioning.

\section{E. Four-Point Probe Measurement Results}

A source-measure instrument (Keithley System SourceMeter 2602) was connected to the four probes through its two channels (a current source and a voltmeter). After contact detection, the probes were positioned at approximately $200 \mathrm{~nm}$ above the sub-

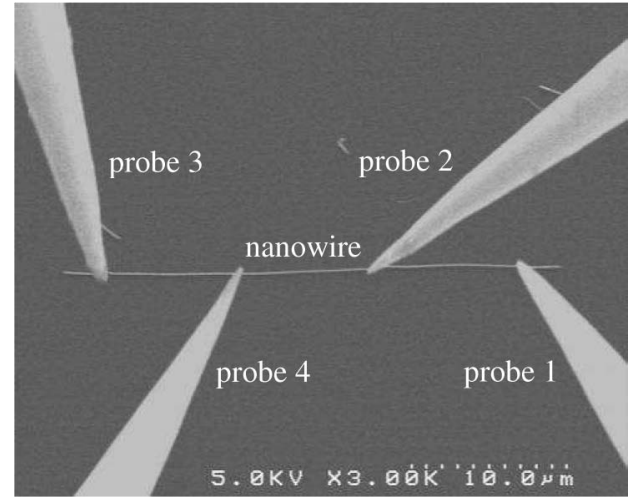

Fig. 10. Four probes landing on target positions for four-point probe measurement.
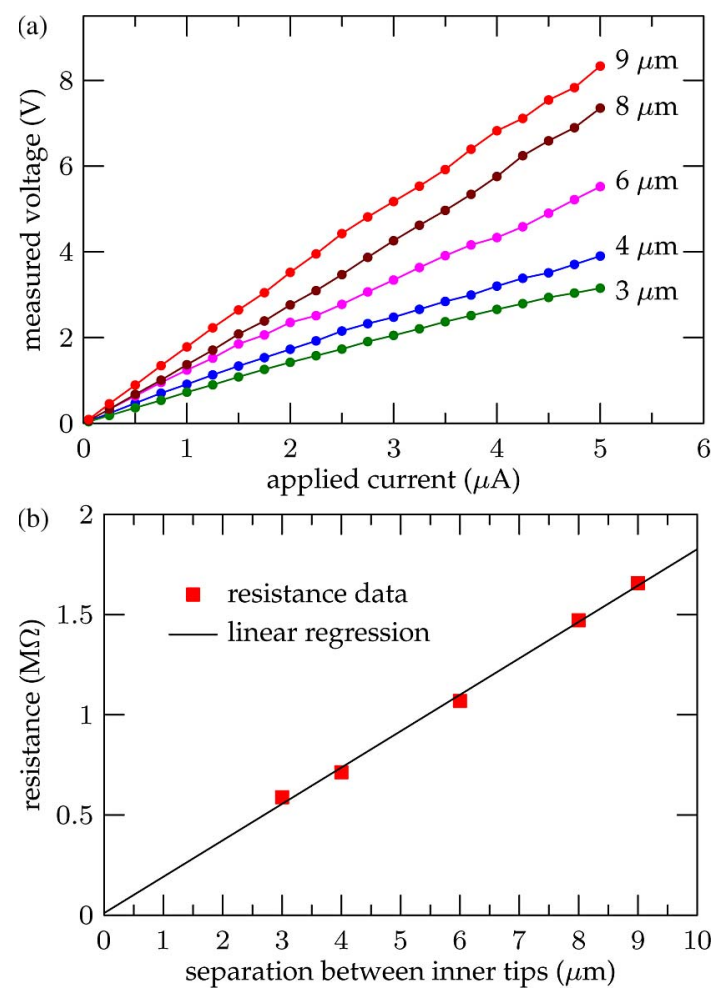

Fig. 11. Four-probe measurement results of a nanowire. (a) $I-V$ data of a nanowire with regard to different separations between the two inner probes. (b) Separation-resistance relationship.

strate, with their corresponding $x y$ coordinate changes (versus the $x y$ coordinate when a probe is in initial contact with the substrate and when a probe is $50 \mathrm{~nm}$ above the substrate) in the image frame recorded.

The system then horizontally servoed the probes to their target positions on a nanowire, taking into account the pixel coordinate changes of the probes from the height of $200 \mathrm{~nm}$ to the target height of $50 \mathrm{~nm}$ above the substrate. A target height of $50 \mathrm{~nm}$ above the substrate was chosen to ensure secured pressing on our nanowires that range from 70 to $100 \mathrm{~nm}$ in diameter. After the probes landed on the nanowire, as shown in Fig. 10, a current sweep was applied to the outer pair of probes (probe 1 and probe 3 ), and the inner pair of probes (probe 2 and probe 4) 
measured the resultant voltages. To quantify the repeatability of this technique, the entire automation process from contact detection to the final $I-V$ measurement were attempted 50 times on five nanowires with $100 \%$ success rate.

The $I-V$ data from a nanowire (83 $\mathrm{nm}$ in width) are shown in Fig. 11 (a). The five data curves correspond to five different separations between the two inner probes. The five corresponding resistance values are proportional to the separations (i.e., the portions of the nanowires between two inner probe tips), as shown in Fig. 11 (b). Assuming the nanowire has a circular cross section, the resistivity was determined to be $9.88 \times$ $10^{-4} \Omega \cdot \mathrm{m}$. When a higher current was applied, and the nanowire finally failed when the current reached $7.4 \mu \mathrm{A}$. The breakdown current density of the nanowire was calculated to be $1.36 \times 10^{9} \mathrm{~A} / \mathrm{m}^{2}$.

\section{CONCLUSION}

This paper demonstrated automated nanoprobing using SEM as a vision sensor. A method for vision-based contact detection was developed to detect the contact between a probe and a substrate for determining relative vertical positions of the probe tip and a nanoobject to be manipulated. A visual servo control system with a feedforward controller was built for closedloop control of multiple nanomanipulators, which demonstrated significant improvement in response time in comparison to the feedforward-controller-free scenario. Four-point probe measurements were conducted on individual tin oxide nanowires lying on a substrate. This technique can also be useful for electrical testing of nanostructures, such as nanodevices comprised of multiple nanoelectrodes and 1-D nanomaterials.

\section{REFERENCES}

[1] M.-F. Yu, M. J. Dyer, G. D. Skidmore, H. W. Rohrs, X. Lu, K. D. Ausman, J. R. V. Ehr, and R. S. Ruoff, "Three-dimensional manipulation of carbon nanotubes under a scanning electron microscope," Nanotechnology, vol. 10, pp. 244-252, 1999

[2] M.-F. Yu, O. Lourie, O. Lourie, K. Moloni, T. F. Kelly, and R. S. Ruoff, "Strength and breaking mechanism of multiwalled carbon nanotubes under tensile load," Science, vol. 287, pp. 637-640, 2000.

[3] T. Fukuda, F. Arai, and L. Dong, "Assembly of nanodevices with carbon nanotubes through nanorobotic manipulations," Proc. IEEE, vol. 91, no. 11, pp. 1803-1818, Nov. 2003.

[4] D. J. Bell, L. Dong, B. J. Nelson, M. Golling, L. Zhang, and D. Grützmacher, "Fabrication and characterization of three-dimensional InGaAs/GaAs nanosprings," Nano Lett., vol. 6, no. 4, pp. 725-729, 2006.

[5] Y. Zhu, F. Xu, Q. Qin, W. Y. Fung, and W. Lu, "Mechanical properties of vapor-liquid-solid synthesized nanowires," Nano Lett., vol. 9, no. 11, pp. 3934-3939, 2009.

[6] A. Lugstein, M. Steinmair, A. Steiger, H. Kosina, and E. Bertagnolli, "Anomalous piezoresistance effect in ultrastrained silicon nanowires," Nano Lett., vol. 10, no. 8, pp. 3204-3208, 2010.

[7] P. A. Williams, S. J. Papadakis, M. R. Falvo, A. M. Patel, M. Sinclair, A. Seeger, A. Helser, and R. M. Taylor II, "Controlled placement of an individual carbon nanotube onto a microelectromechanical structure," Appl. Phys. Lett., vol. 80, pp. 2574-2576, 2002.

[8] Y. Zhu and H. D. Espinosa, "An electromechanical material testing system for in situ electron microscopy and applications," Proc. Nat. Acad. Sci., vol. 102, pp. 14503-14508, 2005.

[9] B. Peng, M. Locascio, P. Zapol, S. Li, S. L. Mielke, G. C. Schatz, and H. D. Espinosa, "Measurements of near-ultimate strength for multiwalled carbon nanotubes and irradiation-induced crosslinking improvements," Nat. Nanotechnol., vol. 3, pp. 626-631, 2008.
[10] R. Agrawal, B. Peng, and H. D. Espinosa, "Experimental-computational investigation of $\mathrm{ZnO}$ nanowires strength and fracture," Nano Lett., vol. 9, pp. 4177-4183, 2009.

[11] D. Zhang, J.-M. Breguet, R. Clavel, V. Sivakov, S. Christiansen, and J. Michler, "In situ electron microscopy mechanical testing of silicon nanowires using electrostatically actuated tensile stages," J. Microelectromech. Syst., vol. 19, pp. 663-674, 2010.

[12] K. Aoki, H. T. Miyazaki, H. Hirayama, K. Inoshita, T. Baba, K. Sakoda, N. Shinya, and Y. Aoyagi, "Microassembly of semiconductor threedimensional photonic crystals," Nat. Mater, vol. 2, pp. 117-121, 2003.

[13] L. Dong, F. Arai, and T. Fukuda, "Destructive constructions of nanostructures with carbon nanotubes through nanorobotic manipulation," IEEE/ASME Trans. Mechatron., vol. 9, pp. 350-357, 2004.

[14] T. Fukuda, M. Nakajima, P. Liu, and H. ElShimy, "Nanofabrication, nanoinstrumentation and nanoassembly by nanorobotic manipulation," Int. J. Robot. Res., vol. 28, pp. 537-547, 2009.

[15] Y. Peng, T. Cullis, and B. Inkson, "Bottom-up nanoconstruction by the welding of individual metallic nanoobjects using nanoscale solder," Nano Lett., vol. 9, pp. 91-96, 2009.

[16] R. T. R. Kumar, S. U. Hassan, O. S. Sukas, V. Eichhorn, F. Krohs, S. Fatikow, and P. Bøggild, "Nanobits: Customizable scanning probe tips," Nanotechnology, vol. 20, pp. 395703-1-395703-6, 2009.

[17] T. Sievers and S. Fatikow, "Real-time object tracking for the robot-based nanohandling in a scanning electron microscope," J. Micromechatron., vol. 3, pp. 267-284, 2006.

[18] S. Fatikow, T. Wich, H. Hülsen, T. Sievers, and M. Jähnisch, "Microrobot system for automatic nanohandling inside a scanning electron microscope," IEEE/ASME Trans. Mechatron., vol. 12, pp. 244-252, 2007.

[19] B. E. Kratochvil, L. Dong, and B. J. Nelson, "Real-time rigid-body visual tracking in a scanning electron microscope," Int. J. Robot. Res., vol. 28, pp. 498-511, 2009.

[20] V. Eichhorn, S. Fatikow, T. Wich, C. Dahmen, T. Sievers, K. N. Andersen, K. Carlson, and P. Bøggild, "Depth-detection methods for microgripper based CNT manipulation in a scanning electron microscope," J. MicroNano Mechatronics, vol. 4, pp. 27-36, 2008.

[21] V. Eichhorn, S. Fatikow, T. Wortmann, C. Stolle, C. Edeler, D. Jasper, O. S. P. Bøggild, G. Boetsch, C. Canales, and R. Clavel, "Nanolab: A nanorobotic system for automated pick-and-place handling and characterization of CNTs," in Proc. IEEE Int. Conf. Robot. Autom., Kobe, Japan, May 2009, pp. 1826-1831.

[22] K. Kim, X. Liu, Y. Zhang, and Y. Sun, "Nanonewton force-controlled manipulation of biological cells using a monolithic MEMS microgripper with two-axis force feedback," J. Micromech. Microeng., vol. 18, p. 055013, 2008

[23] M. Jähnisch and S. Fatikow, "3-D vision feedback for nanohandling monitoring in a scanning electronmicroscope," Int. J. Optomechatronics, vol. 1, pp. 4-26, 2007.

[24] F. Marinello, P. Bariani, E. Savio, A. Horsewell, and L. D. Chiffre, "Critical factors in SEM 3D stereo microscopy," Meas. Sci. Technol., vol. 19, pp. 065705-1-065705-12, 2008.

[25] X. Lin, X. B. He, T. Z. Yang, W. Guo, D. X. Shi, H.-J. Gao, D. D. D. Ma, S. T. Lee, F. Liu, and X. C. Xie, "Intrinsic current-voltage properties of nanowires with four-probe scanning tunneling microscopy: A conductance transition of ZnO nanowire," Appl. Phys. Lett., vol. 89, pp. 043103-1043103-3, 2006.

[26] A. S. Walton, C. S. Allen, K. Critchley, M. . Górzny, J. E. McKendry, R. M. D. Brydson, B. J. Hickey, and S. D. Evans, "Four-probe electrical transport measurements on individual metallic nanowires," Nanotechnol. vol. 18, pp. 065204-1-065204-6, 2007.

[27] T.-H. Kim, X.-G. Zhang, D. M. Nicholson, B. M. Evans, N. S. Kulkarni, B. Radhakrishnan, E. A. Kenik, and A.-P. Li, "Large discrete resistance jump at grain boundary in copper nanowire," Nano Lett., vol. 10, no. 8, pp. 3096-3100, 2010.

[28] R. Lin, M. B. OHansen, R. R. Schlittler, and P. Bøggild, "Micro-fourpoint-probe characterization of nanowires fabricated using the nanostencil technique," Nanotechnology, vol. 15, pp. 1363-1367, 2004.

[29] S. Dohn, K. Mølhave, and P. Bøggild, "Direct measurement of resistance of multiwalled carbon nanotubes using micro four-point probes," Sens. Lett., vol. 3, pp. 1-4, 2005.

[30] S. Yoshimoto, Y. Murata, K. Kubo, K. Tomita, K. Motoyoshi, T. Kimura, H. Okino, R. Hobara, I. Matsuda, S. Honda, M. Katayama, and S. Hasegawa, "Four-point probe resistance measurements using PtIrcoated carbon nanotube tips," Nano Lett., vol. 7, pp. 956-959, 2007.

[31] V. Eichhorn, S. Fatikow, O. S. Sukas, T. M. Hansen, P. Bøggild, and L. G. Occhipinti, "Novel four-point-probe design and nanorobotic dual 
endeffector strategy for electrical characterization of as-grown swcnt bundles," in Proc. IEEE Int. Conf. Robot. Autom., Anchorage, Alaska, May 2010, pp. 4100-4105.

[32] Y. Wang, K.-K. Lew, T.-T. Ho, L. Pan, S. W. Novak, E. C. Dickey, J. M. Redwing, and T. S. Mayer, "Use of phosphine as an n-type dopant source for vapor-liquid-solid growth of silicon nanowires," Nano Lett., vol. 5, pp. 2139-2143, 2005.

[33] S. Valizadeh, M. Abid, F. Hernández-Ramírez, A. R. Rodríguez, K. Hjort, and J. Å. Schweitz, "Template synthesis and forming electrical contacts to single Au nanowires by focused ion beam techniques," Nanotechnology, vol. 17, pp. 1134-1139, 2006.

[34] X. Liu, J. Tong, and Y. Sun, "A millimeter-sized nanomanipulator with sub-nanometer positioning resolution and large force output," Smart Mater. Struct., vol. 16, pp. 1742-1750, 2007.

[35] W. H. Wang, X. Y. Liu, and Y. Sun, "Contact detection in microrobotic manipulation," Int. J. Robot. Res., vol. 26, pp. 821-828, 2007.

[36] L. Sun, C. Ru, W. Rong, L. Chen, and M. Kong, "Tracking control of piezoelectric actuator based on a new mathematical model," J. Micromech. Microeng., vol. 14, pp. 1439-1444, 2004.

[37] C. Ru and L. Sun, "Improving positioning accuracy of piezoelectric actuators by feedforward hysteresis compensation based on a new mathematical model," Rev. Sci. Instrum., vol. 76, pp. 095111-1-095111-8, 2005.

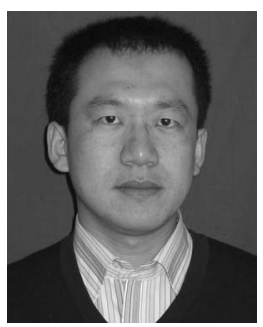

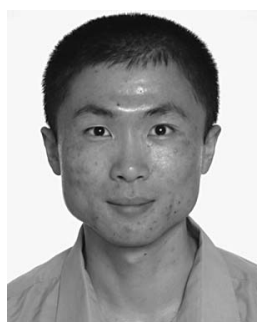

robotics approaches.
Changhai Ru received the Ph.D. degree in mechatronics engineering from Harbin Institute of Technology, Harbin, China, in 2005.

$\mathrm{He}$ is currently a Professor at Robotics and Microsystems Center, Soochow University, China. He was a Postdoctoral Fellow at the Advanced Micro and Nanosystems Laboratory, Department of Mechanical and Industrial Engineering, University of Toronto, ON, Canada. His research interests include micronanomanipulation, nano-positioning technology, and solid-state actuators' driving and control methods.

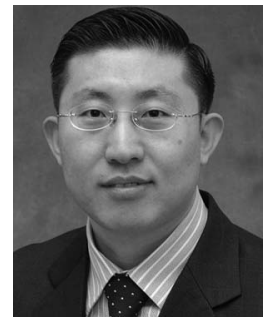

Yu Sun (S'01-M'04-SM'07) received the B.S. degree in electrical engineering from Dalian University of Technology, Dalian, China, in 1996, the M.S. degree from the Institute of Automation, Chinese Academy of Sciences, Beijing, China, in 1999, and the M.S. degree in electrical engineering and the $\mathrm{Ph} . \mathrm{D}$. degree in mechanical engineering from the University of Minnesota, MN, in 2001 and 2003, respectively. He is currently an Associate Professor at the Department of Mechanical and Industrial Engineering and is jointly appointed in the Institute of Biomaterials and Biomedical Engineering and Electrical and Computer Engineering Department, University of Toronto, ON, Canada. He was a Research Scientist at the Swiss Federal Institute of Technology (ETH-Zurich) before joining the Faculty of Toronto in July 2004. He is a McLean Senior Fellow at the University of Toronto and the Canada Research Chair in Micro and Nano Engineering Systems. His research interests include design and fabrication of MEMS/NEMS devices, micro-nanorobotic manipulation under optical and electron microscopes, and manipulation and characterization of biological cells, biomolecules, and nanomaterials.

Dr. Sun is on the Editorial Board of the IEEE Transactions on Automation Science and Engineering, IEEE/ASME Transactions on Mechatronics, and Micro and Nano Letters.

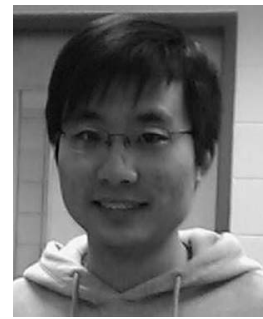

Yu Zhong received the B.S. degree in materials science and engineering from Northeastern University, Shenyang, China, in 2006, and the M.Eng. degree in materials science and engineering from Queen Mary, University of London, London, U.K., in 2007. He is currently working toward the Ph.D. degree at the Nanomaterials Fabrication and Fuel Cell Laboratory, Department of Mechanical and Materials Engineering, University of Western Ontario, ON, Canada.

His research interests include the synthesis of 1-D nanomaterials and their applications in fuel cells.

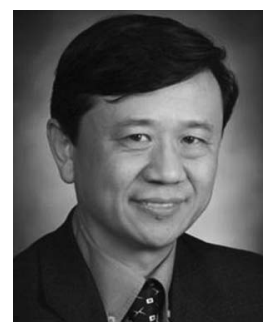

Xueliang Sun received the Ph.D. degree in materials chemistry from the University of Manchester, Manchester, U.K.

He is currently an Associate Professor with the Department of Mechanical and Materials Engineering, University of Western Ontario, London, ON, Canada. $\mathrm{He}$ is the Canada Research Chair in the development of nanomaterials for clean energy. His research activities are currently focused on developing various approaches to synthesize low-dimensional nanomaterials such as carbon nanotubes, semiconducting and Yong Zhang (S'09) received the B.S. and M.S. degrees in mechatronics engineering from Harbin Institute of Technology, Harbin, China, in 2005 and 2007 , respectively. He is currently working toward the Ph.D. degree at the Advanced Micro and Nanosystems Laboratory, Department of Electrical and Computer Engineering, University of Toronto, ON, Canada.

His research interests include the manipulation and characterization of micrometer- and nanometer-sized materials using microelectromechanical systems and metal nanowires, nanoparticles and thin films as well as exploring their applications for energy including fuel cells and Li-ion batteries. He has extensive experience in materials science and engineering, materials chemistry (including electrochemistry) and materials physics.

Prof. Sun is an author of over 60 refereed journal articles and two book chapters. He holds seven U.S. patents related to carbon nanotubes, nanowires, and fuel cells.

David Hoyle, photograph and biography not available at the time of publication.

Ian Cotton, photograph and biography not available at the time of publication. 